\title{
The Effect of Bifidobacterium on Reducing Symptomatic Abdominal Pain in Patients with Irritable Bowel Syndrome: A Systematic Review
}

\author{
Charlotte Pratt ${ }^{1} \cdot$ Matthew D. Campbell ${ }^{1}$ (I) \\ Published online: 18 November 2019 \\ (C) The Author(s) 2019
}

\begin{abstract}
Probiotics, specifically Bifidobacteria, may improve abdominal pain in patients with irritable bowel syndrome (IBS); however, results from randomised controlled trials (RCTs) are conflicting. Here, we systematically reviewed the efficacy of Bifidobacteria on abdominal pain in IBS. We searched MEDLINE, EMBASE and the Cochrane Controlled Trials Register from inception to 20 May 2019, without language or date restrictions. The search strategy comprised of the combination of three concepts: supplementation, abdominal pain, and IBS. Inclusion criteria included double-blind placebo-controlled RCTs featuring Bifidobacteria supplementation in Rome-diagnosed IBS patients. A total of 8 RCTs involving a total of 1045 patients with Rome diagnosed IBS were included. The dose of total Bifidobacteria ranged from $10^{6}$ to $>10^{11}$ cfu (colony-forming unit) and duration of supplementation ranged between 2 and 8 weeks. Bifidobacteria was delivered through either intake of fermented milk products, encapsulation or via a malted milk beverage, with all studies assessing abdominal pain via a visual analogue Likert scale. From the studies included, $50 \%(\mathrm{n}=4)$ of studies found a statistically significant improvement in abdominal pain following Bifidobacteria supplementation compared to placebo, $38 \%(\mathrm{n}=3)$ of studies found non-significant improvements and $12 \%(\mathrm{n}=$ 1) showed a statistically significant dose-response effect of improvement. The evidence shows a heterogeneity of effect for Bifidobacteria dependent upon strain, dosage and delivery method. While not all studies demonstrate significant improvements in abdominal pain, none of the selected studies reported an increase in pain or other adverse effects.
\end{abstract}

Keywords Bifidobacterium $\cdot$ Irritable bowel syndrome $\cdot$ Abdominal pain

\section{Introduction}

Irritable bowel syndrome (IBS) is one of the most widely recognised functional bowel disorders globally, characterised by chronic or recurrent abdominal pain [1]. While it is a common condition, the aetiology is not fully understood. However, classical hallmarks include disturbances in gut microbiota, lowgrade mucosal inflammation, immune activation and altered intestinal permeability [2]. Probiotics, which are live microbial supplements that colonise in the colon and serve to modulate the intestinal microbial-inflammatory-immunological milieu, have been shown to yield beneficial effects on both the clinical course and symptoms of IBS [3, 4]. Probiotics are numerous and exert divergent effects depending upon the unique characteristics of their composition, namely, their genus, species and

Matthew D. Campbell

m.d.campbell@leeds.ac.uk

1 School of Food Science and Nutrition, University of Leeds, Leeds, UK strain [5]; while some probiotics display a desired antiinflammatory effect, others principally impact motility [6] and visceral sensation [7]. Thus, the efficacy of probiotic supplementation on reducing symptoms of IBS is largely dependent upon, and specific to, the individual probiotic used.

Much of the research has investigated the impact of composite probiotic mixtures containing several strains of Lactobacillus, Bifidobacterium and Propionibacterium, which hampers the assessment of specific strains in relieving IBS symptoms. In IBS, patients typically present with significantly lower levels of Bifidobacteria in faecal and duodenal mucosa samples, yet other major bacterial groups remain preserved [4]. Evidence suggests that Bifidobacteria supplementation, which serves to restore a balanced microbial composition, modulates immune function, gut microbiota and intestinal mucosal adhesion in IBS patients [8], with studies demonstrating positive effects on epithelial cell adherence, reinforcement of tight junctions, stimulation of IgA production and cell-mediated immunity, which are impaired in IBS patients $[9,10]$. Based on these promising findings, clinical trials have been conducted to establish whether Bifidobacteria supplementation reduces abdominal pain in IBS; however, 
results are conflicting. Therefore, this investigation aimed to systematically review human studies in which the efficacy of Bifidobacteria supplementation had been examined as a treatment for abdominal pain in IBS.

\section{Methods}

This systematic review was conducted in accordance with PRISMA (Preferred Reporting Items for Systematic Reviews and Meta-analyses) guidelines [11] and prospectively registered. A search of the medical literature was conducted using MEDLINE, EMBASE and the Cochrane Controlled Trials Register from inception to 20 May 2019, without language or date restrictions, for all RCTs investigating the impact of Bifidobacteria on abdominal pain in patients with IBS. The search strategy comprised of the combination of three concepts: supplementation, abdominal pain and IBS. For these three items, relevant keyword variations were used, which included keyword variations in the controlled vocabularies of the different databases, as well as free text word variations. Inclusion criteria included double-blind placebocontrolled RCTs featuring Bifidobacteria supplementation in Rome-diagnosed IBS patients.

Two investigators (CP and MDC) independently reviewed all RCTs by title and abstract and subsequently by full-text evaluation. Any disagreements were resolved through arbitrations with a third researcher independent from the research team. If relevant study information was missing, authors were contacted, and foreign language manuscripts translated where necessary. The bibliographies of all identified studies were used to perform a recursive search of the literature. The quality of the articles was assessed by the two independent reviewers according to the levels of evidence and the recommendations used for good clinical practice [12]. Risk of bias was assessed using the Cochrane Collaboration Risk of Bias tool. Each RCT was given one of three rankings, 'high risk', 'low risk', or 'unknown risk', in each of the following domains: sequence generation, allocation concealment, blinding of participants and personnel, blinding of outcome assessors, incomplete data, selective outcome reporting, and other sources of bias. Discrepancies which arose during this process were resolved firstly by discussion then by a third independent researcher where necessary. Risk of bias outcomes is presented within Supplement 1, Table 1.

\section{Results}

In total, 343 records were identified through database screening, of these 8 RCTs were eligible and included in this review (Fig. 1; Table 1). A total of 1045 adults aged between 20 and 75 years with Rome-diagnosed IBS were included. The ranges of doses of total Bifidobacteria were $10^{6}$ to $>10^{11} \mathrm{cfu}$ and duration of supplementation between 2 and 8 weeks. Bifidobacteria was delivered through either intake of fermented milk products $(n=3)[13,16,17]$, encapsulation $(n=4)[10,14,15,18]$, or via a malted milk beverage $(n=1)$ [6], with all studies assessing abdominal pain via a visual analogue Likert scale.

From the studies included, $50 \%(n=4)$ of studies found a statistically significant improvement in abdominal pain following Bifidobacteria supplementation compared to placebo, $38 \%(\mathrm{n}=3)$ of studies found non-significant improvements, and $12 \%(\mathrm{n}=1)$ showed a statistically significant doseresponse effect of improvement (Table 1).

\section{Enriched Fermented Milk Products}

Agrawal and colleagues [13] investigated trialled Bifidobacterium lactis in a fermented milk product consumed daily over a 4-week intervention compared to a daily probiotic-naive placebo non-fermented product. Individuals under the treatment condition were found to have significantly lower levels of abdominal pain compared to the control group. Guyonnet and colleagues [16] investigated the effect of Bifidobacterium animalis in a yoghurt compared to a placebo heat-treated yoghurt. Abdominal pain improved equally in both the treatment and placebo group overtime at 3 and 6 weeks compared to baseline; no conditional differences between treatment and placebo were evident. Similarly, Min and colleagues [17] investigated the use of daily intake of yoghurt enriched with Bifidobacterium animalis subspecies lactis. This placebo-controlled trial compared symptoms of abdominal pain following an 8 -week treatment phase and failed to yield statistically significant benefits. Of note, the placebo in this trial consisted of a traditional yoghurt, which is likely to contain traces of Bifidobacteria, albeit in significantly lower amounts, which may account for the null effects on abdominal pain between the two conditions. Furthermore, the interventional treatment contained acacia gum, a complex indigestible polysaccharide fermented in the colon, which may confound potential improvements in abdominal pain resulting from Bifidobacteria alone.

\section{Encapsulation}

Guglielmetti and colleagues [15] trialled daily encapsulated delivery of Bifidobacterium bifidum against a placebo control over a 4 -week treatment period. The authors observed significant time-course reductions in subjective abdominal pain from weeks 1 to 4 , which persisted into the subsequent 2-week washout period. Charbonneau et al. [14] investigated the effect of encapsulated delivery of daily Bifidobacterium longum subspecies infantis over an 8-week period and reported reduced levels of 


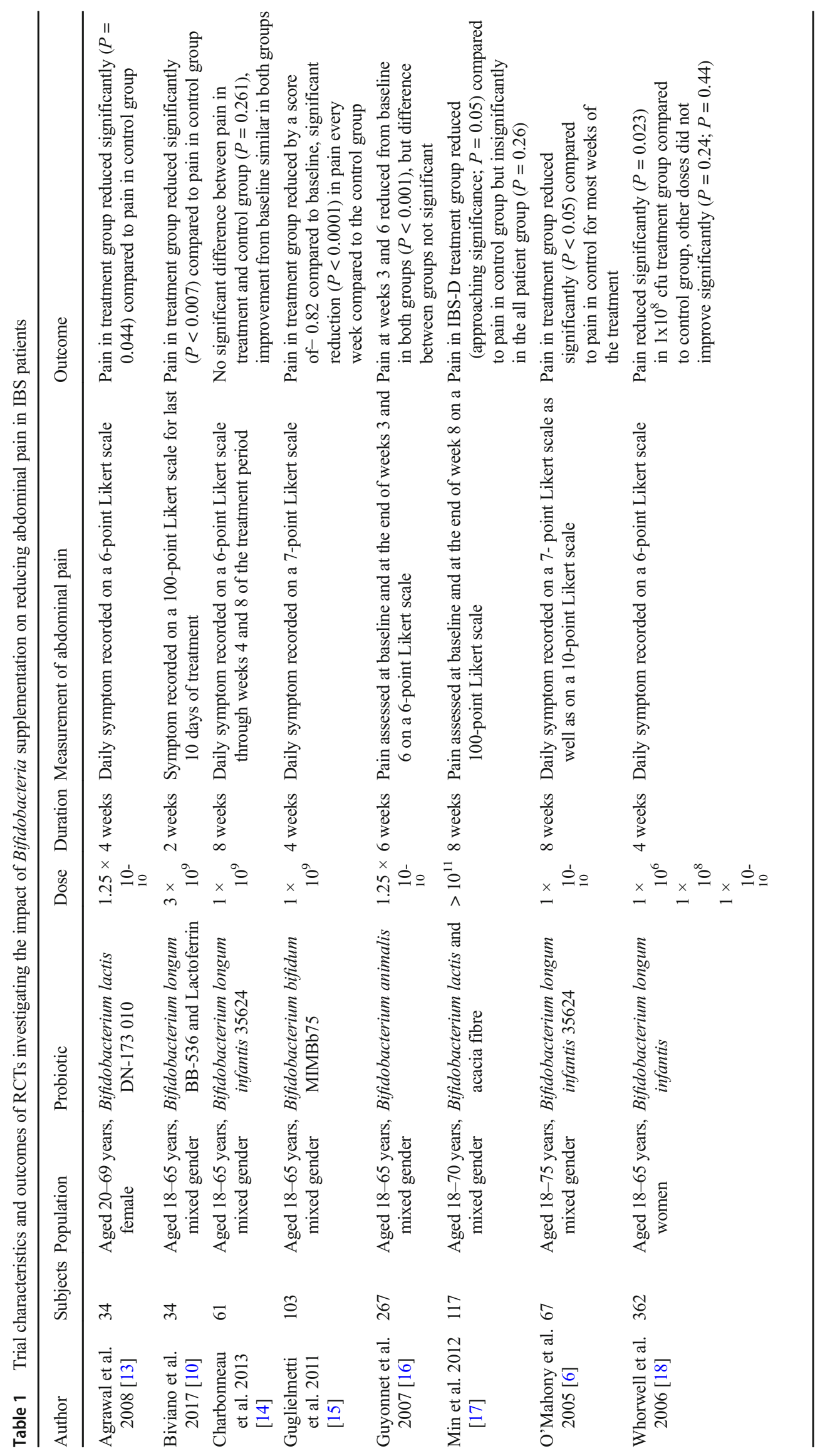


Fig. 1 Flowchart of trial selection
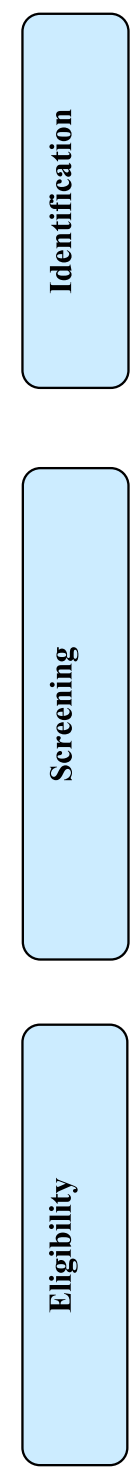

perceived abdominal pain at the end of the supplementation period. Similarly, these findings are supported by those of Biviano et al. [10] in which 2-week daily supplementation of Bifidobacterium longum and Lactoferrin was trialled, resulting in a significant reduction in pain scores in the treatment group compared to control. Of note, this study included Lactoferrin, a prebiotic for Bifidobacterium into the treatment product; further research is needed to establish whether the Lactoferrin in addition to Bifidobacterium in isolation carries any additive effect on abdominal pain or adjunct effects on other symptoms of IBS. Whorwell et al. [18] investigated the dose-response of encapsulated Bifidobacterium infantis supplementation whereby subjects were randomly allocated to receiving either a daily capsule of Bifidobacterium infantis at a dosage of $1 \times 10^{6}, 1 \times 10^{8}$ or $1 \times 10^{10} \mathrm{cfu}$ or placebo control for 4 weeks. The authors reported a

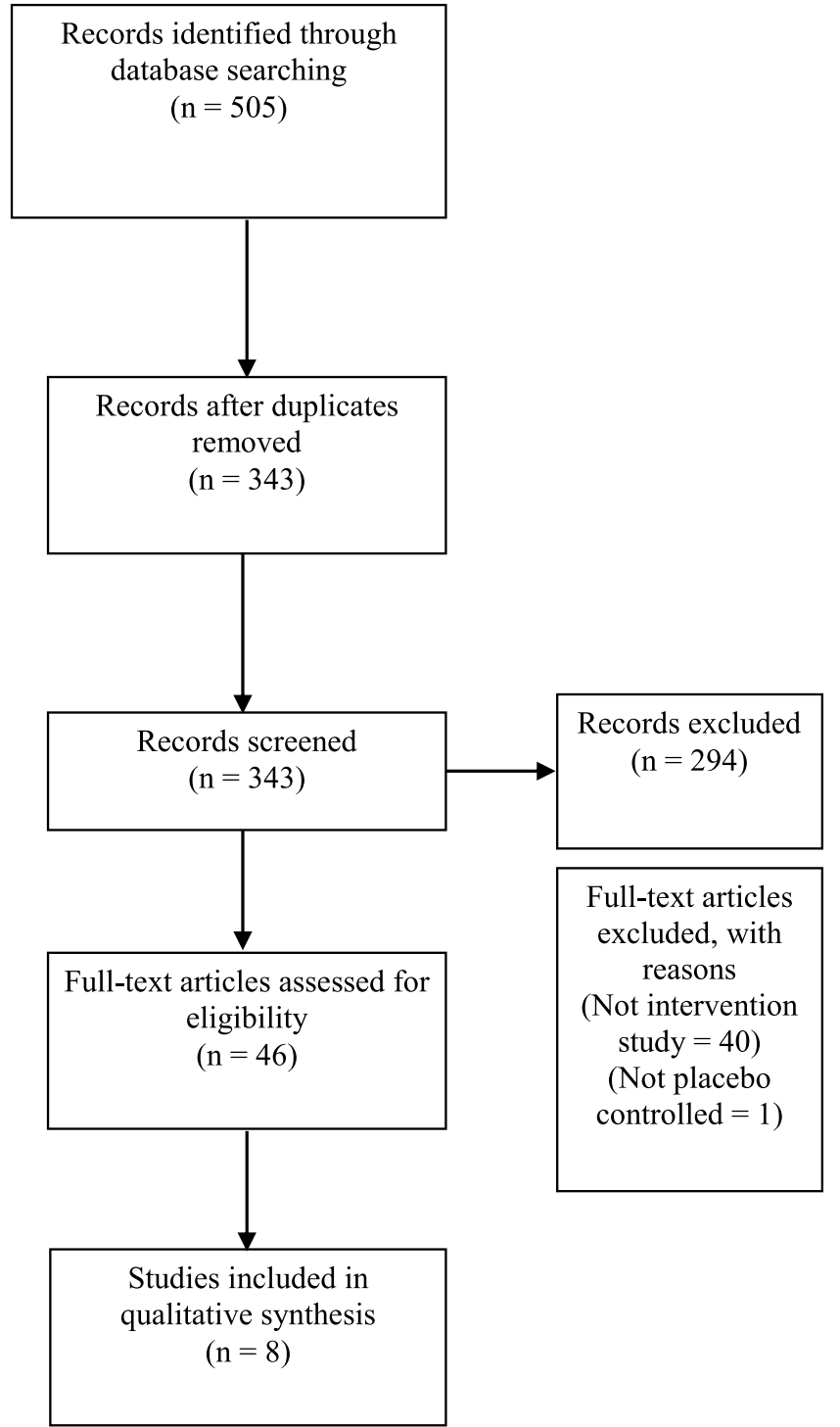

beneficial treatment effect for Bifidobacterium infantis at a dosage of $1 \times 10^{8} \mathrm{cfu}$ which persisted throughout the subsequent two-week washout period, while dosages of 1 $\times 10^{6}$ or $1 \times 10^{10} \mathrm{cfu}$ did not yield significant reductions in abdominal pain.

\section{Malted Milk Beverage}

O'Mahony and colleagues [6] investigated the impact of Bifidobacteria infantis on abdominal pain delivered through a daily malted milk beverage over a course of 8 weeks. Significant time course improvements were observed following treatment, with improvements in abdominal pain evident at week-1, peaking at week-2, with a sustained suppression of symptoms up to trial cessation at 8 weeks. 


\section{Discussion}

There is conflicting evidence regarding the clinical utility of probiotics, specifically Bifidobacteria, in reducing subjective abdominal pain in patients with IBS. Our systematic review provides the most comprehensive and contemporary review to date assessing the impact of Bifidobacteria on abdominal pain in patients with IBS. Considering the cumulative findings from 8 RCTs with a total of 1045 adults with IBS, we conclude that evidence shows a heterogeneity of effect for Bifidobacteria dependent upon strain, dosage and delivery method. Importantly, while not all studies demonstrate a statistically significant improvement in abdominal pain, none of the selected studies in this review reported an increase in pain or other adverse effects compared to placebo.

Irritable bowel syndrome (IBS) is one of the most widely recognised functional bowel disorders globally, characterised by chronic or recurrent abdominal pain [1]. In the UK, $\sim 17 \%$ of the general population live with IBS, and up to $50 \%$ of all general practitioner (GP) visits for gastrointestinal disturbances relate to IBS symptoms [19]. IBS poses a significant personal, societal and economic burden [20-22], thus clinically effective and pragmatic treatment options which serve to reduce the symptoms of IBS are much needed. Although several reviews have previously concluded that probiotics improve IBS symptoms including abdominal pain [23-28], many reviews feature studies investigating the use of composite probiotic mixtures containing several strains of Lactobacillus, Bifidobacterium and Propionibacterium, which hampers the assessment of specific strains in relieving IBS symptoms. As such, this is the first study to systematically review the clinical effectiveness of single strains of Bifidobacterium on symptomatic abdominal pain in patients with IBS. Despite applying stringent inclusion criteria and rigorous methodology, there are several concerns regarding the quality of available evidence. Firstly, the available literature lacks uniformity in the Bifidobacterium species tested and the dosage used. From the investigations reviewed in this study, three differing strains of Bifidobacteria were sampled, and dosages varied from $10^{6}$ to $>10^{11} \mathrm{cfu}$. Secondly, the delivery of the probiotics varied between studies, including delivery via fermented milk products $[13,16,17]$, a malted milk beverage [6] or via encapsulation [10, 14, 15, 18]. Thirdly, treatment periods varied from 2 weeks to 8 weeks with variable washout periods; considering the intermittent nature of symptomatic IBS abdominal pain, short treatment durations and short observation windows may fail to adequately capture the effect, and legacy effect, of supplementation or severity of symptoms. Fourthly, the composition of placebos used in some studies may confound study findings $[10,17]$. In future studies, it would be helpful to stratify IBS patients by clinical presentation and/or by severity of symptoms, and include longer treatment durations and longer wash- out periods to establish long-term legacy effects and whether dosages can be taken less frequently.

Currently, the evidence shows a heterogeneity of effect for Bifidobacteria on abdominal pain in IBS dependent upon strain, dosage and delivery method. Future research should consider investigating whether divergent treatment responses are determined by supplementation characteristics (strain, dosage and delivery method), as well as individual clinical parameters which may influence treatment effectiveness (e.g. gut microbiota composition, gut motility), and whether these clinical parameters are useful tools for predicting treatment outcomes. This review should help others obtaining a balanced view of the relevant literature available. Presently, it is difficult to strongly advocate the use of Bifidobacteria for reducing abdominal pain in IBS; however, as none of the selected studies in this review reported an increase in pain or other adverse effects, we can at least have a greater degree of confidence in remarking that Bifidobacteria supplementation is unlikely to have an adverse effect.

Author Contributions CP and MDC designed the protocol, performed the searches, independently screened all records identified through database searches, assessed risk, synthesised data and prepared the manuscript. Both authors revised the final version of the manuscript and have read and approved the final manuscript.

Funding Information This study was funded by the School of Food Science and Nutrition, University of Leeds.

\section{Compliance with Ethical Standards}

Competing Interests The authors declare that they have no competing interests.

Open Access This article is distributed under the terms of the Creative Commons Attribution 4.0 International License (http:// creativecommons.org/licenses/by/4.0/), which permits unrestricted use, distribution, and reproduction in any medium, provided you give appropriate credit to the original author(s) and the source, provide a link to the Creative Commons license, and indicate if changes were made.

\section{References}

1. Lovell RM, Ford AC (2012) Global prevalence of and risk factors for irritable bowel syndrome: a meta-analysis. Clin Gastroenterol Hepatol 10:712-721

2. Holtmann GJ, Ford AC, Talley NJ (2016) Pathophysiology of irritable bowel syndrome. Lancet Gastroenterol Hepatol 1:133-146

3. Fanigliulo L, Comparato G, Aragona G, Cavallaro L, Iori V, Maino M, Cavestro GM, Soliani P, Sianesi M, Franzè A, di Mario F (2006) Role of gut microflora and probiotic effects in the irritable bowel syndrome. Acta Biomed 77:85-89

4. Kerckhoffs AP, Samsom M, van der Rest ME, de Vogel J, Knol J, Ben-Amor K, Akkermans LM (2009) Lower bifidobacteria counts in both duodenal mucosa-associated and fecal microbiota in irritable bowel syndrome patients. World J Gastroenterol 15:2887-2892 
5. Furrie E (2005) Is Bifidobacterium a more effective probiotic therapy than lactobacillus for patients with irritable bowel syndrome? Nat Clin Pract Gastroenterol Hepatol 2:304-305

6. O'Mahony L, McCarthy J, Kelly P et al (2005) Lactobacillus and bifidobacterium in irritable bowel syndrome: symptom responses and relationship to cytokine profiles. Gastroenterology. 128:541551

7. Verdú EF, Bercík P, Bergonzelli GE, Huang XX, Blennerhasset P, Rochat F, Fiaux M, Mansourian R, Corthésy-Theulaz I, Collins SM (2004) Lactobacillus paracasei normalizes muscle hypercontractility in a murine model of postinfective gut dysfunction. Gastroenterology. 127:826-837

8. Angelakis E, Merhej V, Raoult D (2013) Related actions of probiotics and antibiotics on gut microbiota and weight modification. Lancet Infect Dis 13:889-899

9. Ford AC, Quigley EMM, Lacy BE et al (2014) Efficacy of prebiotics, probiotics and synbiotics in irritable bowel syndrome and chronic idiopathic constipation: systematic review and meta-analysis. Am J Gastroenterol 109:1547-1561

10. Biviano I, Rossi S, Piacentino D et al (2017) Effect of bifidobacterium longum Bb536 plus lactoferrin in the treatment of irritable bowel syndrome. A double blind clinical trial. Adv Res Gastroenterol Hepatol 6:ARGH.MS.ID.555691

11. Moher D, Liberati A, Tetzlaff J et al (2009) Preferred reporting items for systematic reviews and meta-analyses: the PRISMA statement. PLoS Med 6:e1000097

12. Moher D, Schulz KF, Altman DG (2001) The CONSORT statement: revised recommendations for improving the quality of reports of parallel-group randomised trials. Lancet. 357:1191-1194

13. Agrawal A, Houghton LA, Morris J, Reilly B, Guyonnet D, Goupil Feuillerat N, Schlumberger A, Jakob S, Whorwell PJ (2009) Clinical trial: the effects of a fermented milk product containing bifidobacterium lactis $D N-173010$ on abdominal distension and gastrointestinal transit in irritable bowel syndrome with constipation. Aliment Pharmacol Ther 29:104-114

14. Charbonneau D, Gibb RD, Quigley EMM (2013) Fecal excretion of bifidobacterium infantis 35624 and changes in fecal microbiota after eight weeks of oral supplementation with encapsulated probiotic. Gut Microbes 4:201-211

15. Guglielmetti S, Mora D, Gschwender M, Popp K (2011) Randomised clinical trial: bifidobacterium bifidum MIMBb75 significantly alleviates irritable bowel syndrome and improves quality of life - a double-blind, placebo-controlled study. Aliment Pharmacol Ther 33:1123-1132

16. Guyonnet D, Chassany O, Ducrotte P, Picard C, Mouret M, Mercier $\mathrm{CH}$, Matuchansky C (2007) Effect of a fermented milk containing bifidobacterium animalis $D N-173010$ on the health-related quality of life and symptoms in irritable bowel syndrome in adults in primary care: a multicentre, randomized, double-blind, controlled trial. Aliment Pharmacol Ther 26:475-486

17. Min YW, Park SU, Jang YS, Kim YH, Rhee PL, Ko SH, Joo N, Kim SI, Kim CH, Chang DK (2012) Effect of composite yogurt enriched with acacia fiber and bifidobacterium lactis. World J Gastroenterol 18:4563-4569

18. Whorwell PJ, Altringer L, Morel J, Bond Y, Charbonneau D, O'Mahony L, Kiely B, Shanahan F, Quigley EM (2006) Efficacy of an encapsulated probiotic bifidobacterium infantis 35624 in women with irritable bowel syndrome. Am J Gastroenterol 101: $1581-1590$

19. Khanbhai A, Sura DS (2013) Irritable bowel syndrome for primary care physicians. Br J Med Pract 6:a608

20. Thompson WG, Heaton KW, Smyth GT, Smyth C (2000) Irritable bowel syndrome in general practice: prevalence, characteristics, and referral. Gut. 46:78-82

21. Gunn MC, Cavin AA (2003) Management of irritable bowel syndrome. Postgrad Med J 79:154-158

22. Dai C, Zheng C-Q, Jiang M, Ma XY, Jiang LJ (2013) Probiotics and irritable bowel syndrome. World J Gastroenterol 19:5973-5980

23. Brenner DM, Moeller MJ, Chey WD et al (2009) The utility of probiotics in the treatment of irritable bowel syndrome: A systematic review. Am J Gastroenterol 104:1033-1049

24. McFarland LV, Dublin S (2008) Meta-analysis of probiotics for the treatment of irritable bowel syndrome. World J Gastroenterol 14: 2650-2661

25. Hoveyda N, Heneghan C, Mahtani KR et al (2009) A systematic review and meta-analysis: probiotics in the treatment of irritable bowel syndrome. BMC Gastroenterol BioMed Central 9:15

26. Nikfar S, Rahimi R, Rahimi F, Derakhshani S, Abdollahi M (2008) Efficacy of probiotics in irritable bowel syndrome: a meta-analysis of randomized, controlled trials. Dis Colon Rectum 51:1775-1780

27. Moayyedi P, Ford AC, Talley NJ, Cremonini F, Foxx-Orenstein AE, Brandt LJ, Quigley EM (2010) The efficacy of probiotics in the treatment of irritable bowel syndrome: a systematic review. Gut. 59:325-332

28. Didari T, Mozaffari S, Nikfar S, Abdollahi M (2015) Effectiveness of probiotics in irritable bowel syndrome: updated systematic review with meta-analysis. World J Gastroenterol 21:3072-3084

Publisher's Note Springer Nature remains neutral with regard to jurisdictional claims in published maps and institutional affiliations. 